\title{
Internet User Stickiness in the Social Q \& A Community from the Perspective of Interactivity - A Case Study on Zhihu Website
}

\author{
Sheng-jun YUAN and Jia-xin $\mathrm{CHEN}^{1}$ \\ School of Business, Guilin University of Electronic Technology, Guilin 541004, China
}

\begin{abstract}
This paper mainly explores how the user's perceptual interaction towards a specific website affects the user stickiness and the interaction mechanism of various influencing factors of user stickiness, which may encourage socialized Q \& A community operators to adopt more appropriate ways to enhance user's stickiness. We comprehensively use the planning behavior theory and technology acceptance model with the perceptual perspective of interaction. Based on the framework of "belief $\rightarrow$ attitude $\rightarrow$ intention", an attempt is made to construct a conceptual model of influencing factors of user stickiness in socialized Q \& A community by collecting the data through questionnaire survey, and using spss 23.0 and amos 23.0 to analyze the data and validate the model. The empirical research results prove that the factors such as perceived usefulness, perceived ease of use, information exchange perception, social interaction perception satisfaction, and heart flow experience have positive effects on social users. We conclude that the operators can build and consolidate user engagement, attract and retain the users by enhancing interactive perception, optimizing user experience, and improving their satisfaction.
\end{abstract}

Keywords. Interactivity, social Q \& A community, user stickiness, Zhihu website

\section{Introduction}

The social Q \& A community is a system, in which users can ask questions, answer and evaluate in the process of interaction[1-3]. At present, the representative of domestic socialized Q \& A community development is Zhihu website, which was established in 2011.The Q \& A community in China, taking Zhihu as an example, is developing extensively with the spread of Internet. Though the survey shows that by 2019, the number of registered Zhihu has reached 200 million, but the data reveals that most of the registered users are silent and one-time consumers. On an average, less than $40 \%$ of active users visit Zhihu more than 7 times a week, with low user stickiness and low continuous participation. The core purpose of socialized Q \& A community is to generate knowledge through continuous interaction to meet users' access to information and create value [4] (Wang Wei 2018).

\footnotetext{
${ }^{1}$ Corresponding Author, Jia-xin CHEN, Guilin University of Electronic Technology, Guilin 541004, China; Email:954743805@qq.com
} 
The fundamental way to establish and consolidate user stickiness is to improve users' interactive perception. Only some scholars built the comprehensive planning behavior theory based on the technology acceptance model to establish the integrated use model (UTAUT) [5] and other researchers added the Expectation Confirmation Theory on the basis of the integrated model to put forward the information system continuous use model (ecm-it) [6]. But the obvious problem of these two models is that they ignore the impact of interpersonal interaction on stickiness in interactive perception and the role of factors such as flow experience. This paper aims (1) to integrate the factors affecting user stickiness from the perspective of interactivity, (2) to test how the influencing factors interact and, (3) which factors play a leading role? This study will more comprehensively explain the influencing factors of user stickiness, and help the operators to improve their existing strategies to enhance user stickiness.

\section{Research design and hypothesis}

\subsection{The establishment of research model}

Review of relevant literature reveals that the perceptual interaction is a multidimensional construct, and its dimensions are divided into different standards. In fact, the process of users participating in the social Q \& A community is a combination of "human interaction with the system" and "interaction between people". Therefore, we divide the interaction into two forms: human-machine interaction and interpersonal interaction, from the perspective of user participation by are using the division method of Hoffman [7]. According to the technology acceptance model, we divide humanmachine interaction into perceived ease of use and perceived usefulness [8].

In 2000, Kurniawan [9] proposed a model of influencing factors of customer stickiness of the network retailer. This model takes community involvement, website attractiveness and convenience as prepositional variables whereas, pleasure and customer satisfaction are the intermediate variables and customer stickiness is a result variable. This model verified that five predictive variables had a significant impact on customer stickiness. Based on this, an attempt is made here to formulate a conceptual model of the influence factors of the community user stickiness by taking the socialized Q \& A community as a research object, combining its characteristics and comprehensively using the interactive theory, the technical acceptance model, and the heart flow experience theory.

\subsection{Research hypotheses}

(1) Interactive perception and satisfaction

For the social Q \& A community, the community will achieve high satisfaction when it perceives that the community is easy to use and can get quick help [10]. Accordingly, in the social Q \& A community, we put forward the following hypothesis:

$\mathrm{H} 1 \mathrm{a}$ : Perceived ease of use has a positive impact on satisfaction.

H1b: Perceived usefulness has a positive impact on satisfaction.

A successful social Q \& A community not only promotes the user's continuous information interaction in the community, but also helps users to establish social relations network in the process of interaction, using the platform to constantly carry out effective 
information transmission and emotional interflow thereby, promoting the user satisfaction Therefore, in the social Q \& A community, we put forward the following hypothesis:

$\mathrm{H} 2 \mathrm{a}$ : Information exchange perception has a positive impact on satisfaction.

$\mathrm{H} 2 \mathrm{~b}$ : Social interaction perception has a positive impact on satisfaction.

(2) Interactive perception and the heart flow experience

The experience of heart flow refers to the overall feeling of an individual fully invested in a certain activity.. Whether individuals can enter the heart flow experience or not depends on skills and challenges. Skill is a kind of ability for individuals to successfully complete given tasks whereas, the challenge means all the efforts required to accomplish the task. Accordingly, in the social Q\&A community, we put forward the hypothesis that:

H3a: Perceived ease of use has a positive impact on the flow of heart experience

$\mathrm{H} 3 \mathrm{~b}$ : Perceived usefulness has a positive impact on the flow of heart experience

In the background of social Q\&A community, there are few studies on the effects of interpersonal interaction on cardiac experience. However, the positive relationship between interpersonal interaction and heart flow experience has been verified many times in other contexts, such as tourism community, online learning platform, and online game. This paper extends the conclusion to the social Q\&A community. Accordingly, we put forward the hypothesis:

$\mathrm{H} 4 \mathrm{a}$ : Information exchange perception has a positive impact on the flow of heart experience;

H4b: Social interaction perception has a positive impact on the flow of heart experience.

The heart flow experience can attract individuals and significantly affect their subsequent attitudes and behaviors. Users may expect to experience positive experiences in social Q\&A communities, and they will be satisfied when expectations are confirmed, which is confirmed in the background of online banking, sports website, and mobile shopping, and so on. Accordingly, in the social Q \& A community, we put forward the hypothesis that:

H5: The heart flow experience positively affects satisfaction

When individuals enter the heart flow experience and reach the optimal state, they may immerse themselves in it and spend a lot of time digging deep into the community. When expecting this experience again, the frequency of return visits will increase. One of the characteristics of user stickiness is user's continuous use behavior. Accordingly, in the social Q\&A community, we put forward the hypothesis that:

H6: The heart flow experience positively affects user stickiness

When users have positive attitude and overall attachment to the contents, functions, products and services in the system, stickiness is bound to produce. Satisfaction is a powerful determinant of sustained behavior, and is the best valuation of relationship strength in long-term relationship marketing. Many studies in the internet background have shown that user satisfaction is an important driving force for stickiness. Accordingly, in the social Q \& A community, we put forward the hypothesis that:

H7: Satisfaction positively affects user stickiness. 


\section{Research Methods}

\subsection{Questionnaire Design and Collection}

The measurements of the related variables in this essay are mainly cited from maturity scales at home and abroad, and are adjusted according to the research contents and China's national conditions. Seven potential variables are considered. The final questionnaire comprises of two parts.

The questionnaire was spread in some places including Guangxi province, Guangdong Province, Beijing, Shanghai, and Sichuan province. Totally, 430 questionnaires were collected including the invalid ones comprising of incomplete filling, non-subscribers from Zhihu community and complete in all respect. 372 effective questionnaires were collected with an efficiency rate is $86.5 \%$.

It is observed that Cronbach's $\alpha$ and Combinatorial Reliability (CR values) of every dimension in this scale is close to or higher than 0.8 , which satisfy the demand of reliability. The standard loading of every latent variable are all higher than $0.7, \mathrm{CR}$ values are higher than 0.8 and the average variance extracted (AVE) are higher than 0.8, confirming that the scales have a good convergent validity. From the result, the square roots (diagonal) of the AVE of every latent variable are higher than the correlation coefficients among the latent variables and others, which shows that the distinctiveness among every latent variable is pretty strong, and the discriminant validity of the model is good.

\subsection{Reliability \& Validity of data}

The analysis was mainly by SPSS23.0 and AMOS23.0. We used Exploratory Factor Analysis (EFA) and Confirmatory Factor Analysis for the reliability and validity verification.

Firstly, we did KMO test and Bartlett Test of Sphericity to the valid samples to confirm that the questionnaire have construct validity. The result showed that the KMO value is 0.847 , higher than 0.5 , the chi-square value of Bartlett Test of Sphericity is 4459.009 , reaching the significant level $(\mathrm{p}=0.000<0.001)$, indicating that the samples are suitable for factor analysis.

Secondly, we carried out the reliability and validity verification from the index including standard loading of every dimension, combinatorial validity, combinatorial reliability, average variances extracted, etc. It is observed that Cronbach's $\alpha$ and Combinatorial Reliability (CR values) of every dimension in this scale is close to or higher than 0.8 , which satisfy the demand of reliability. The standard loading of every latent variable are all higher than 0.7 , CR values are higher than 0.8 and the average variance extracted (AVE) are higher than 0.8, confirming that the scales have a good convergent validity. The result shows that the square roots (diagonal) of the AVE of every latent variable are higher than the correlation coefficients among the latent variables and others, which shows that the distinctiveness among every latent variable is pretty strong, and the discriminant validity of the model is good.

\subsection{Model Amendment}

After reliability and validity verification of the questionnaire, we used AMOS23.0 to verify the hypothetical model. Putting the data of every latent variable into the model 
to have the first fitting, we found that the regression coefficient of social interaction perception and flow experience is 0.08 , the $t$ value is 0.145 , and the $P$ value is higher than 0.1 revealing that the social interaction perception has no direct influence on the flow experience. Then we deleted path $\mathrm{H} 4 \mathrm{~b}$, and carried out a small adjustment to the residual relationship.

We did the goodness-of-fit analysis of the amended model by fitting every index of confirmatory factor. The results reveal that CMIN/DF is less than 3, PGFI is more than 0.5 , index like GFI, AGFI, IFI, TLI, CFI are all higher than 0.8, and RMSEA is smaller than 0.08 , showing that all meet the relevant standard. In general, the confirmatory factor analysis model fits well. The model and data also fit well. The subsequent analysis was also tested on the basis of the revised model.

\subsection{Hypothetical Test}

The results shows that the hypotheses $\mathrm{H} 1 \mathrm{a}, \mathrm{H} 1 \mathrm{~b}, \mathrm{H} 2 \mathrm{a}, \mathrm{H} 2 \mathrm{~b}$ are tested. Also from the perspective of the influencing factors of flow experience, the effects range from high to low for perceived usefulness, perceived ease of use, and information exchange perception $(0.317>0.226>0.135)$, respectively. Thus, hypothesis $\mathrm{H} 3 \mathrm{a}, \mathrm{H} 3 \mathrm{~b}$ and $\mathrm{H} 4 \mathrm{a}$ are tested. Flow experience positively affects the satisfaction $(0.151)$ hence, hypothesis H5 is also tested. Similarly, satisfaction influences more to user stickiness than the flow experience $(0.390>0.261)$, so hypothesis H6 and H7 are tested.

\section{Research conclusions and Counter measures}

\subsection{Research conclusions}

The results show that the four dimensions of interactive perception have a significant positive impact on satisfaction, with the greatest effect on perceived usefulness. This means that in the social Q \& A community, the user's biggest motivation is to use his own instrumental demand, which is consistent with the product positioning of the Q \& A community. Secondly, the impact of perceived ease of use on satisfaction is higher than that of information interaction perception and social interaction perception. The influence of social interaction perception on satisfaction is lower than that of the information exchange, indicating that users are more inclined to exchange information in the community. Information search, browsing and effective response enhance satisfaction. Social interaction perception has a significant positive impact on satisfaction, indicating that interpersonal and emotional communication between like-minded users in the community has a positive impact on satisfaction.

The study of the heart flow experience found that the perceived usefulness has a greater impact over perceived ease of use, which corroborates with the literature. As one of the dimensions of interpersonal interaction perception, information exchange perception has a positive impact on the experience of heart flow. In the context of the social Q \& A community, the frequent exchange of information between users, especially the exchange of hidden information, strengthens the depth and breadth of information cognition, resulting in immersion and the state of experience. In addition, research shows that user stickiness is the result variable of satisfaction and heart flow experience, and heart flow experience positively affects user satisfaction. 


\subsection{Counter measures}

At present, the socialized Q \& A community is still in the development stage. The empirical results of this paper have a certain guiding role for the marketing practice activities of community operators. We strongly trust that through these results community operators should enhance users' continuous access intention and increase users' stickiness by adopting the following strategies:

(1) Based on the data, we believe that the first thing operators should do is to build the usefulness of the community and focusing on improving the information quality. To solve these problems operators can establish an external control mechanism to control the response quality, integrate internal and external resources, build an expert team, improve professionalism, review existing information through various means and eliminate the useless information.

(2) Secondly, we believe that operators should improve the quality of information system, optimize interface design through easy construction, simplify user operation and enhance user feedback mechanism according to the users' differentiated needs. In addition, when new functions appear in the product, they should be quickly offered to the user to ensure rapid mastery.

(3) Finally, we believe that operators should pay attention to develop users' interpersonal interaction and improve users' perception of information exchange. To improve users' perception of social interaction, operators should, on the one hand, establish cooperative relations with other platforms and expand users' original relationship network; on the other hand, on the basis of protecting users' information security, encourage users' information disclosure and promote the interaction of users in the same industry and across industries.

\section{References}

[1] Morris M R, Teevan J, Panovich K. What do people ask their social networks, and why?: a survey study of status message q \& a behavior [C]. International Conference on Human Factors in Computing Systems. 2010 Jan;62(1):1739-1748.

[2] Kang M, Kim B, Gloor P, et al. Understanding the effect of social networks on user behaviors in community-driven knowledge services [J]. Journal of the Association for Information Science \& Technology. 2011 'Jan;62(6): 1066-1074.

[3] Gazan R. Social Q \& A [J]. Journal of the Association for Information Science \& Technology. 2011 Mar;62(12): 2301-2312.

[4] Shah C, Oh S, Oh J S. Research agenda for social Q \& A is [J]. Library \& Information Science Research,.2009 May;31(4): 205-209.

[5] Shah C, Kitzie V. Social Q \& A and virtual reference - comparing apples and oranges with the help of experts and users [J]. Journal of the Association for Information Science \& Technology. $2012 \mathrm{Jul}$; 63(10):2020-2036.

[6] Chenxiaoyu, Deng shengli, Sun yameng. Progress and Prospects of User Information Behavior Research in Social Question and Answering Communities [J]. Documentation, Information \& Knowledge. 2015 Jan;2(4):71-81.

[7] Hoffman D L, Novak T P. Marketing in hypermedia computer-mediated environments: Conceptual foundations [J]. Journal of Marketing. 1996 Oct; 60(3): 50-68.

[8] Davis, Fred D, Bagozzi, et al. User acceptance of computer technology: a comparison of two theoretical models [J]. Management Science.1989 May;35(8): 982-1003.

[9] Kurniawan S H. Modeling Online Retailer Customer Preference and Stickiness: A Mediated Structural Equation Model [C]. Pacific Asia Conference on Information It \& society. 2000 Apr;20(8): 238-252.

[10] Bhattacherjee A. Understanding Information Systems Continuance: An Expectation-Confirmation Model [J]. Mis Quarterly. 2001 Sept;25(3): 351-370. 